MINIREVIEW

\title{
Modulation of the MAP kinase signaling cascade by Raf kinase inhibitory protein
}

\author{
Nicholas TRAKUL, Marsha R. ROSNER* \\ Ben May Institute for Cancer Research, University of Chicago, 5841 S. Maryland Avenue., Chicago, IL 60637, USA
}

\begin{abstract}
Proteins like Raf kinase inhibitory protein (RKIP) that serve as modulators of signaling pathways, either by promoting or inhibiting the formation of productive signaling complexes through protein-protein interactions, have been demonstrated to play an increasingly important role in a number of cell types and organisms. These proteins have been implicated in development as well as the progression of cancer. RKIP is a particularly interesting regulator, as it is a highly conserved, ubiquitously expressed protein that has been shown to play a role in growth and differentiation in a number of organisms and can regulate multiple signaling pathways. RKIP is also the first MAP kinase signaling modulator to be identified as playing a role in cancer metastasis, and identification of the mechanism by which it regulates Raf-1 activation provides new targets for therapeutic intervention.
\end{abstract}

Keywords: MAPK, RKIP, Raf, PKC.

\section{INTRODUCTION}

One of the key challenges in signal transduction research is to delineate the mechanisms by which common signaling cascades are utilized by different external stimuli to induce discrete cellular outputs. The MAP kinase cascade is an evolutionarily conserved signaling module that is activated by a diverse set of signals and that stimulates numerous biological processes including growth and differentiation. The pathway consists of a MAP kinase kinase kinase (MKKK) that phosphorylates and activates a MAP kinase kinase, which in turn phosphorylates the TXY activation domain of MAP kinase (reviewed in [1]). The first characterized subfamily of MAP kinases, termed extracellular signal regulated kinases (ERKs), are activated by growth factors and other stimuli via a cascade involving Ras, Raf-1 kinase, and MEK/ERK kinase (MEK).

Activation of MAP kinase is under exquisite regulatory control, particularly at the level of Raf- 1 activation. The $\mathrm{N}$-terminal regulatory domain of Raf- 1 interacts with Ras leading to membrane association, dephosphorylation at negative regulatory sites, conformational changes to expose the kinase domain, and subsequent phospho-

*Correspondence: Marsha Rich ROSNER

Tel: +773-702-0380;

E-mail: m-rosner@uchicago.edu rylation at activating sites such as serine 338 (S338) and tyrosine 341 (Y341) (reviewed in [2]). The activation of the MAPKKK Raf is an intricate, multistep process that is still not completely understood. The complexity of Raf regulation, coupled with its position in the MAPK pathway, indicate that it should be the major point of regulatory control.

\section{THE MAPK PATHWAY AND RAF ACTIVATION}

There are three isoforms of Raf in mammals, A-Raf, BRaf, and Raf-1 (or c-Raf). A and B-Raf show specific patterns of tissue expression, whereas Raf- 1 is more widely expressed. All the Raf isoforms can be divided into two domains, the amino terminal regulatory domain, which contains two conserved regions (CR1 and CR2), and the carboxy terminal kinase domain, which contains the third conserved region, CR3 [3].

The process of Raf activation is very complex, with multiple phosphorylations and dephosphorylations occurring that ultimately lead to a conformational shift whereupon the inhibition of the $\mathrm{N}$-terminal regulatory domain is relieved. The most important activating phosphorylations occur in the kinase domain of Raf- 1 at Serines 338 and tyrosines 340 and 341 , as these are the primary residues phosphorylated in response to oncogenes and growth factors. It has been shown that the p21-activated kinase (Pak) is capable of phosphorylating Raf-1 specifically on 
Serine 338 [4]. Tyrosines 340 and 341 are phosphorylated by the Src family of kinases [5]. Replacement of any of these amino acids with non-phosphorylatable residues impairs the activation of Raf, while substitution of 338 with phosphomimetic negatively charged residues restores regulation by Src. Substitution of tyrosines 340 and 341 with acidic residues results in constitutive Raf activity, suggesting a hierarchal relationship among these residues. Other important sites of activating phosphorylation occur within the region known as the activation loop, on T491 and S494. Substitution of these residues severely impairs Raf activity, while substituting phosphomimetic residues at these sites can increase basal activity. B-Raf contains a phosphomimetic aspartate in place of the corresponding residue for Y341, and appears to be constitutively phosphorylated on the S388 equivalent site. This could explain B-Raf's higher basal activity and its higher specific activity towards MEK as compared to Raf-1 [6]. PKCs have also been shown to be capable of phosphorylating Raf, specifically on Serines 497 and 499, but mutation of these sites has no effect on Raf activity [7].

Inactive Raf is found in a multiprotein complex of around $300-500 \mathrm{kD}$, and more than 30 Raf interacting proteins have been reported in the literature [2], indicating that there could be many possible Raf regulators. These include 14-3-3, a phospho-serine specific binding adapter protein, which modulates the activity of many signaling proteins. Other reported Raf interacting proteins include: Hsp90, which is required for Raf activity, the phosphatases PP2A and Cdc25; other kinases, including Akt, PKCs, Jak, the Src familiy tyrosine kinases, and the MAPK ERK5; adapter proteins other than 14-3-3, such as SUR-8, CNK, KSR and Grb10, the Sprouty family of proteins, and Raf kinase inhibitory protein (RKIP).

\section{RKIP}

RKIP was originally purified from bovine brain extracts and named phosphatidylethanolamine-binding protein (PEBP), based on its ability to preferentially bind this particular class of phospholipids [8]. Subsequent studies revealed that PEBP/RKIP is a widely expressed and highly conserved protein that does not share any significant homology with any known protein family, with homologs in, E.Coli, A.thaliana, S.cerevisae, C.elegans, and D. melanogaster, which display a high degree of interspecies similarity [9].

Crystal structures for human [10], bovine [11], bacterial [12], and plant [13], as well as mouse PEBP-2 [14] RKIP have been solved. These studies have shown that all homologs display a similar, novel topology, characterized by a large central $\beta$-sheet flanked by a smaller $\beta$-sheet with a two carboxy-terminal $\alpha$-helices. There exists a highly conserved phosphate-binding pocket within the structure that has been shown to be critical to RKIP/PEBP function. The carboxy-helices may play a role in regulating access to the binding pocket. Since RKIP/PEBP has shown the capacity to bind phospholipids and nucleotides, it has been postulated that it could function as a lipid transporter in membrane biogenesis or as a G protein. However, analysis of the structure of PEBP/RKIP revealed that the binding pocket was much too shallow to accommodate a lipid tail, and the structure has no similarity to known Gproteins [11] suggesting that the binding pocket could be utilized to bind phospho-proteins. Experiments examining the interaction of PEBP/RKIP with model membranes found that there was no significant binding to phosphatidylethanolamine containing membranes, and that instead, RKIP bound negatively charged membranes in an electrostatic manner, suggesting that RKIP does not use phosphatidylethanolamine binding as a mechanism to localize to the plasma membrane [15].

Numerous functions of PEBP/RKIP proteins have been reported. In yeast, the one RKIP homolog (Tfs $1 \mathrm{p}$ for "Twenty-Five suppressor") has been shown to be an inhibitor of carboxypeptidase Y, a vacuolar enzyme [16] as well as a specific inhibitor of Ira2p, a yeast Ras GAP whose function is to attenuate Ras activation by causing hydrolysis of GTP. TFS mutant strains exhibited enhanced resistance to heat shock, suggesting that there was a higher level of Ras activity. It should be noted that the effect was subtle, which the authors explained by the inability of Tfs $1 p$ to inhibit the closely related Ras-GAP Ira1p.

In nematodes, RKIP was found to be part of membranous material shedded from the surface of cells, and hypothesized to play a role in protection from host immune detection. In Drosophila, which expresses at least 7 RKIP isoforms, RKIP/PEBPs were hypothesized to play a role in odorant binding, as the proteins are localized in antennae and secreted into the lumen of olfactory hairs [9].

In plants, PEBP/RKIP has been associated with the control of the commitment to shoot growth or inflorescence (flowering). This represents a key step in the switch from the vegetative to reproductive growth phases in plants, and may be considered somewhat analogous to the decision of growth versus differentiation in mammalian cells. Mutations in plant RKIP homologs, including centroradialis (CEN) in Antirrhinum, terminal flower-1 (TF-1) in Arabidopsis, and self-pruning (SP) in tomato, result in plants with limited shoot growth and branching, which is an agriculturally useful mutation in tomato plants, as it facilitates mechanical harvesting [17]. In an example supporting the similarity between plant and mammalian RKIP, a yeast two-hybrid assay using SP identified a number of 
binding partners including a serine/threonine kinase (called SPAK) which, like Raf-1, possesses dual 14-3-3 binding sites, and shows sequence similarity to the NIMA like family of mammalian kinases, which play a role in the process of mitosis.

In addition to the wide variety of functions attributed to RKIP in lower organisms, mammalian RKIP has also been described to have functions other than that of a regulator of MAPK signaling. RKIP/PEBP has been shown to inhibit serine proteases, specifically thrombin, neuropsin, and chymotrypsin, although it shares no homology to any of the known families of serine protease inhibitors. It was also reported that, despite having no obvious secretion signal, RKIP/PEBP can be immunolocalized on the cell surface and detected in conditioned media [18].

Previous to its characterization as a MAPK inhibitor, the most extensive body of work on RKIP/PEBP focused on its function as a source of a neurostimulatory peptide (reviewed in [19]). Amino acids 2-12 of RKIP/PEBP are identical to the Hippocampal cholinergic neurostimulatory peptide (HCNP), a factor that was purified based on its ability to stimulate acetylcholine (Ach) synthesis in hippocampal explant cultures. Release of HCNP appears to be mediated through NMDA receptor signaling [19]. An enzyme that is capable of cleaving RKIP/PEBP to form HCNP has been detected in rat hippocampal extracts, but this enzyme has not been positively identified to date.

Aberrations in HCNP or its precursor protein, PEBP/ RKIP, have been noticed in some neuropathologies. In sections taken from Alzheimer's patients, antibodies directed against the HCNP portion of RKIP show distinct staining in Hirano Bodies, which have been demonstrated to be increased in both Alzheimer's and Parkinson's patients. Also, it has been noted that some patients with Alzheimer's exhibit increased levels of HCNP in their cerebral spinal fluid (CSF) [20]. Additionally, in senescence-accelerated-prone mice, which demonstrate an increased age-accelerated deterioration in the ability to learn, brain sections showed both an increase in HCNP, and the mRNA for its precursor [21].

Another tissue in which PEBP/RKIP has been studied is the testis. It was found that PEBP was an abundant secretory product of testicular germ cells [22]. Also, rodents possess an RKIP isoform, PEBP-2, whose expression appears to be testis-specific [23].

\section{RKIP AS AN ENDOGENOUS INHIBITOR OF THE MAPK PATHWAY}

Yeung and coworkers, who identified RKIP as a protein that interacts with the kinase domain of Raf-1, first demonstrated that RKIP acts as an inhibitor of MAPK signaling [24]. Removal of RKIP by microinjection of anti-RKIP antibodies or through antisense activated an AP-1 reporter. Conversely, overexpression of RKIP suppressed transcription from the AP-1 promoter. RKIP overexpression was additionally able to suppress transformation induced by a constitutively active Raf. Since the authors had shown that RKIP could interact with Raf, as well as MEK and ERK, the other components of the MAPK pathway, they next attempted to determine at which step in the MAPK pathway RKIP was acting. RKIP was unable to inhibit AP-1 activation by a constitutively active MEK. In a later paper [7], the authors showed that RKIP could decrease the binding of Raf-1 to MEK, while the interaction between MEK and ERK was unaffected. The binding sites for MEK and Raf-1 overlap in RKIP, with both overlapping in the phosphatidylethanolamine-binding motif, supporting the idea that RKIP cannot bind to MEK and Raf-1 simultaneously. Although overexpressed RKIP can inhibit Raf-1 phosphorylation of MEK, recent studies from our laboratory indicate that endogenous RKIP regulates activation of Raf-1 rather than the interaction between MEK and Raf-1.

It has also been suggested [25] that RKIP could be involved in regulation of the NF- $\mathrm{KB}$ pathway. Inhibition of RKIP enhanced NF- $\mathrm{KB}$ transcription, while overexpression of RKIP reduced basal transcription levels. RKIP was also shown to interact with upstream kinase activators of IKK, such as TAK and NIK, and inhibit the phosphorylation of IKK in response to TNF $\alpha$. These data suggest that RKIP can inhibit signaling pathways other than ERK, acting in a similar fashion (i.e. at the MAPKKK/MAPKK level).

In addition to the ERK and NF- $\kappa B$ pathway, RKIP has been implicated as playing a role in GPCR signaling. It was noted that RKIP could facilitate the activation of opioid or somatastation receptors by an undescribed mechanism [26]. Later, it was found that RKIP could regulate G-protein coupled receptor kinase-2 (GRK2), which may explain its role in GPCR signaling [27]. GRK-2 functions to phosphorylate GPCRs, uncoupling them from their associated G-proteins and targeting them for endocytosis and degradation. It was found that RKIP is able to bind to the amino terminus of GRK-2 and inhibit its ability to phosphorylate GPCRs. Mutation of the PKC phosphorylation site, Serine 153, which was originally identified as a trigger for RKIP release from Raf-1 [28], abrogated RKIP's ability to bind and inhibit GRK-2, suggesting that phosphorylation of RKIP decreases its affinity for Raf while increasing its affinity for GRK-2, which was confirmed by co-immunoprecipitations of Raf, GRK-2, and RKIP from a number of cell lines and tissues using a variety of stimuli. This work establishes a role for RKIP as an inhibitor with the ability to switch from one pathway to another dependent on its phosphorylation state, and is the first such example reported. 
A link between RKIP and cancer was supplied when it was determined that a metastatic prostate cancer cell line (C42-B) expressed lower RKIP levels than the non-metastatic cell line from which it was derived (LnCaP) [29]. Using immunocytochemistry to assess RKIP levels in prostate tissue, it was found that benign tissue expressed the highest levels of RKIP, cancerous tissue expressed significantly less, and that RKIP expression was lowest in metastatic tissue. Overexpression or reduction of RKIP in the C42-B cell line had no effect on either cell growth, or the ability to grow in soft agar. However, modulating RKIP levels did have an effect on in vitro invasion assays. In vivo, metastatic cells that overexpressed RKIP showed a marked decrease in the number of mice that developed metastases (70\%). Additionally, it was noted that the vasculature in the primary tumors of RKIP overexpressing cells was less developed, suggesting that RKIP could function as a metastasis suppressor by decreasing angiogenesis and vascular invasion.

Recent evidence indicates that RKIP plays an important role in cell survival. RKIP was shown to potentiate the action of chemotherapeutic agents [30]. Thus, ectopic expression of RKIP in cancer cells sensitized cells to drug induced apoptosis, and downregulation of RKIP conferred resistance to chemotherapy. RKIP exhibited effects on both the ERK pathway as well as NF- $\kappa \mathrm{B}$ signaling, and restoration of either pathway was able to rescue the apoptotic sensitive phenotype of RKIP overexpressing cells. One possible explanation for the role of RKIP in prostate cancer and as a potentiator of chemotherapeutic agents comes from recent results from our laboratory suggesting that RKIP modulates the mitotic checkpoint. Thus, loss of RKIP could lead to an increase in chromosomal abnormalities and genetic changes, ultimately enhancing the metastatic potential of tumorigenic cells.

\section{THE ROLE OF PKCS IN MITOGENESIS AND DIFFERENTIATION OF RAT NEURONAL PRE- CURSORS}

Raf can be activated by Protein Kinase C (PKC), and one mechanism involves the release of RKIP-mediated inhibition [31]. PKCs are a multigene family of serine/ threonine kinases comprised of 13 known isozymes, divided into three classes, based on their calcium or phospholipid dependence for activation. The classical (or conventional) PKCs, which include PKC $\alpha, \beta \mathrm{I}, \beta \mathrm{II}$, and $\gamma$, require both calcium and phospholipid for activation. The novel PKCs, which include PKC $\delta, \varepsilon, \eta, \theta$, and $v, \mu$, require phospholipid, but are calcium independent. Finally, the atypical $\mathrm{PKC} \zeta, 1$, and $\lambda$ are both calcium and phoshpholipid independent. While the PKCs are structurally similar, they vary widely in cellular and tissue distribution. They also vary widely in their physiological effects, causing migration, proliferation, differentiation, or apoptosis depending on the cell system used, and have been implicated in the activation of numerous pathways, including the MAPK, NF- $\kappa \mathrm{B}, \mathrm{Wnt}$, as well as caspase signaling cascades [32].

We have previously shown [33] that EGF, which is a mitogenic factor, but not FGF, which is a neurotrophic factor, in the SV40 conditionally immortalized cell rat embryonic hippocampal cell line H19-7, is capable of activating $\mathrm{PKC} \zeta$, and this activation is dependent on $\mathrm{PI} 3 \mathrm{~K}$. Elimination of $\mathrm{PKC} \zeta$ through the use of antisense nucleotides, a dominant negative $\mathrm{PKC} \zeta(\mathrm{PKC} \zeta \mathrm{KR})$, or a peptide pseudosubstrate specific for $\mathrm{PKC} \zeta$ abrogated ERK activation in response to EGF. Activation of the upstream activator of ERK, MEK, was also inhibited by these approaches. Proliferation of H19-7 cells in response to EGF, as assessed by BrdU incorporation, was also inhibited by blocking $\mathrm{PKC} \zeta$.

Conversely, it was also found that the $\mathrm{PKC} \delta$ isoform is necessary for MAPK activation downstream of the neurogenic factor FGF, as treatment with the specific PKC $\delta$ inhibitor Rottlerin inhibited activation of ERK in response to FGF. This effect could also be produced by elimination of PKC $\delta$ via antisense. In the H19-7 system, PKC $\delta$ seems to be acting somewhere between Raf and MEK, as Rottlerin has no effect on Raf activation, but does inhibit the phosphorylation of MEK in response to FGF [34]. These findings led us to postulate that there is a hypothetical PKC $\zeta$ target, at or upstream of Raf-1 which mediates MAPK activation and cellular proliferation through the EGF receptor, and a target for $\mathrm{PKC} \delta$ between Raf and MEK. PKCs can phosphorylate Raf-1 and MEK directly, but this phosphorylation is weak, and occurs at sites that do not potentiate the activity of these kinases. We therefore considered the possibility that Raf- 1 regulatory proteins might be potential substrates of PKC and identified Raf Kinase Inhibitory Protein (RKIP) known as PEBP, as a key target.

These studies led to the identification of a mechanism for regulating RKIP's role as an endogenous inhibitor of MAP kinase signaling [28]. PKC, activated by either phorbol esters or epidermal growth factor (EGF), can phosphorylate RKIP at S153, and this phosphorylation causes the dissociation of RKIP from Raf-1 and subsequent activation of the MAPK pathway. Recent results from our laboratory have also elucidated the mechanism by which RKIP suppresses MAP kinase signaling. We demonstrated that RKIP inhibits activation of Raf-1 by blocking activating phosphorylations of residues on Raf-1. Furthermore, the regulation of RKIP is shown to be growth factor-specific in at least two cell systems, providing a new mechanism for the selective activation of the MAP kinase signaling 
cascade leading to discrete physiological outputs.

\section{REFERENCES}

1 Pearson G, Robinson F, Beers Gibson, et al. Mitogen-activated protein (MAP) kinase pathways: regulation and physiological functions. Endocr Rev 2001; 22:153-83.

2 Kolch W. Meaningful relationships: the regulation of the Ras/ Raf/MEK/ERK pathway by protein interactions. Biochem J 2000; 351 Pt 2:289-305.

3 Morrison DK, Cutler RE. The complexity of Raf-1 regulation. Curr Opin Cell Biol 1997; 9:174-9.

4 King AJ, Sun H, Diaz B, et al. The protein kinase Pak3 positively regulates Raf-1 activity through phosphorylation of serine 338. Nature 1998; 396:180-3.

5 Morrison DK, Heidecker G, Rapp UR, Copeland TD. Identification of the major phosphorylation sites of the Raf-1 kinase. J Biol Chem 1993; 268:17309-16.

6 O'Neill E, Kolch W. Conferring specificity on the ubiquitous Raf/MEK signalling pathway. Br J Cancer 2004; 90:283-8.

7 Yip-Schneider MT, Miao W, Lin A, et al. Regulation of the Raf1 kinase domain by phosphorylation and 14-3-3 association. Biochem J 2000; 351:151-9.

8 Bernier I, Tresca JP, Jolles P. Ligand-binding studies with a 23 $\mathrm{kDa}$ protein purified from bovine brain cytosol. Biochim Biophys Acta 1998; 871:19-23.

9 Frayne J, Ingram C, Love S, Hall L. Localisation of phosphatidylethanolamine-binding protein in the brain and other tissues of the rat. Cell Tissue Res 1999; 298:415-23.

10 Banfield MJ, Barker JJ, Perry AC, Brady RL. Function from structure? The crystal structure of human phosphatidylethanolamine-binding protein suggests a role in membrane signal transduction. Structure 1998; 6:1245-54.

11 Serre L, Vallee B, Bureaud N, Schoentgen F, Zelwer C. Crystal structure of the phosphatidylethanolamine-binding protein from bovine brain: a novel structural class of phospholipid-binding proteins. Structure 1998; 6:1255-65.

12 Serre L, Pereira de Jesus K, Zelwer C, et al. Crystal structures of YBHB and YBCL from Escherichia coli, two bacterial homologues to a Raf kinase inhibitor protein. J Mol Biol 2001; 310: 617-34.

13 Banfield MJ, Barker JJ, Perry AC, Brady RL. Function from structure? The crystal structure of human phosphatidylethanolamine-binding protein suggests a role in membrane signal transduction. Structure 1998; 6:1245-54.

14 Simister PC, Banfield MJ, Brady RL. The crystal structure of PEBP-2, a homologue of the PEBP/RKIP family. Acta Crystallogr D Biol Crystallogr 2002; 58:1077-80.

15 Vallee BS, Tauc P, Brochon JC, et al. Behaviour of bovine phosphatidylethanolamine-binding protein with model membranes. Evidence of affinity for negatively charged membranes. Eur J Biochem 2001; 268:5831-41.

16 Bruun AW, Svendsen I, Sorensen SO, Kielland-Brandt MC, Winther JR. A high-affinity inhibitor of yeast carboxypeptidase $\mathrm{Y}$ is encoded by TFS1 and shows homology to a family of lipid binding proteins. Biochemistry 1998; 37:3351-7.

17 Pnueli L, Gutfinger T, Hareven D, et al. Tomato SP-interacting proteins define a conserved signaling system that regulates shoot architecture and flowering. Plant Cell 2001; 13:2687-702.

18 Hengst U, Albrecht H, Hess D, Monard D. The phosphatidylethanolamine-binding protein is the prototype of a novel family of serine protease inhibitors. J Biol Chem 2001; 276:535-40.

19 Ojika K, Mitake S, Tohdoh N, et al. Hippocampal cholinergic neurostimulating peptides (HCNP). Prog Neurobiol 2000; 60: 37-83.

20 Tsugu Y, Ojika K, Matsukawa N, et al. High levels of hippocampal cholinergic neurostimulating peptide (HCNP) in the CSF of some patients with Alzheimer's disease. Eur J Neurol 1998; 5:561-9.

21 Matsukawa N, Tooyama I, Kimura H, et al. Increased expression of hippocampal cholinergic neurostimulating peptiderelated components and their messenger RNAs in the hippocampus of aged senescence-accelerated mice. Neuroscience 1999; 88:79-92.

22 Saunders PT, McKinnell C, Millar MR, et al. Phosphatidylethanolamine binding protein is an abundant secretory product of haploid testicular germ cells in the rat. Mol Cell Endocrinol 1995; 107:221-30.

23 Hickox DM, Gibbs G, Morrison JR, et al. Identification of a novel testis-specific member of the phosphatidylethanolamine binding protein family, pebp-2. Biol Reprod 2002; 67:917-27.

24 Yeung K, Seitz T, Li S, et al. Suppression of Raf-1 kinase activity and MAP kinase signalling by RKIP. Nature 1999; 401:173-7.

25 Yeung KC, Rose DW, Dhillon AS, et al. Raf kinase inhibitor protein interacts with NF-kappaB-inducing kinase and TAK1 and inhibits NF-kappaB activation. Mol Cell Biol 2001; 21: 7207-17.

26 Kroslak T, Koch T, Kahl E, Hollt V. Human phosphatidylethanolamine-binding protein facilitates heterotrimeric $\mathrm{G}$ proteindependent signaling. J Biol Chem 2001; 276:39772-8.

27 Lorenz K, Lohse MJ, Quitterer U. Protein kinase C switches the Raf kinase inhibitor from Raf-1 to GRK-2. Nature 2003; 426: 574-9.

28 Corbit KC, Trakul N, Eves EM, et al. Activation of Raf-1 signaling by protein kinase $\mathrm{C}$ through a mechanism involving $\mathrm{Raf}$ kinase inhibitory protein. J Biol Chem 2003; 278:13061-8.

$29 \mathrm{Fu} \mathrm{Z}$, Smith PC, Zhang L, et al. Effects of raf kinase inhibitor protein expression on suppression of prostate cancer metastasis. J Natl Cancer Inst 2003; 95:878-89.

30 Chatterjee D, Bai Y, Wang Z, et al. RKIP sensitizes prostate and breast cancer cells to drug-induced apoptosis. J Biol Chem 2004; 279:17515-23.

31 Corbit KC, Trakul N, Eves EM, et al. Activation of Raf-1 signaling by protein kinase $\mathrm{C}$ through a mechanism involving $\mathrm{Raf}$ kinase inhibitory protein. J Biol Chem 2003; 278:13061-8.

32 Dempsey EC, Newton AC, Mochly-Rosen D, et al. Protein kinase $\mathrm{C}$ isozymes and the regulation of diverse cell responses. Am J Physiol Lung Cell Mol Physiol 2000; 279:L429-38.

33 Corbit KC, Soh JW, Yoshida K, et al. Different protein kinase C isoforms determine growth factor specificity in neuronal cells. Mol Cell Biol 2000; 20:5392-403.

34 Corbit KC, Foster DA, Rosner MR. Protein kinase Cdelta mediates neurogenic but not mitogenic activation of mitogenactivated protein kinase in neuronal cells. Mol Cell Biol 1999; 19:4209-18. 